\title{
Enquête
}

Archives de la revue Enquête

3 | 1996

Interpréter, Surinterpréter

\section{Risquer l'interprétation}

Pertinences interprétatives et surinterprétations en sciences sociales

Risking interpretation. Interpretative and overinterpretative relevance in social sciences

\section{Bernard Lahire}

\section{(2) OpenEdition \\ Journals}

Édition électronique

URL : http://journals.openedition.org/enquete/373

DOI : 10.4000/enquete.373

ISSN : 1953-809X

Éditeur :

Cercom, Éditions Parenthèses

Édition imprimée

Date de publication : 1 novembre 1996

Pagination : 61-87

\section{Référence électronique}

Bernard Lahire, «Risquer l'interprétation », Enquête [En ligne], 3 | 1996, mis en ligne le 11 juillet 2013 , consulté le 19 avril 2019. URL : http://journals.openedition.org/enquete/373 ; DOI : 10.4000/ enquete.373

Ce document a été généré automatiquement le 19 avril 2019 


\section{Risquer l'interprétation}

Pertinences interprétatives et surinterprétations en sciences sociales

Risking interpretation. Interpretative and overinterpretative relevance in social sciences

\section{Bernard Lahire}

1 À entendre les propos ordinaires des chercheurs en sciences sociales, il semble parfois en aller en matière d'interprétations comme en matière de goûts et de couleurs : chacun pourrait revendiquer le droit de posséder sa propre interprétation des faits et cette interprétation personnelle ne saurait finalement se discuter. Celui qui prétendrait vouloir examiner la valeur heuristique ou le bien-fondé empirique d'une interprétation apparaitrait dès lors comme un ennemi de la démocratie interprétative et des droits fondamentaux de l'homme de science à proposer son interprétation. En définitive, lorsqu'un chercheur en appelle à l'irréductible droit à la différence interprétative comme à un droit qui n'entraînerait aucun devoir théorique, méthodologique ou empirique, alors le terme "interprétation » constitue une manière d'éviter l'affrontement des objections et de clore prématurément le débat scientifique, une façon en tout cas d'ouvrir la voie à toutes les formes d'indifférentisme scientifique.

2 Pourtant chaque chercheur a éprouvé le sentiment, en lisant des textes scientifiques, qu'il existe sur le marché réel (et non idéalisé ou restreint au sous-marché des produits les plus «purs») de la production scientifique des interprétations plausibles, fortes ou convaincantes et d'autres qui ne le sont pas ou qui le sont moins.

3 Cette impression ne cesse en premier lieu d'être éveillée par la lecture des travaux de recherche réalisés par des étudiants apprentis sociologues, apprentis historiens ou apprentis anthropologues qu'aucun enseignant-chercheur - par devoir professionnel - ne se prive de corriger. Les jugements, parfois sévères, portés dans les marges des mémoires de recherche ne cessent de souligner les imprudences interprétatives ou les interprétations "à côté de la plaque", maladroites, mal étayées, insuffisamment argumentées ou empiriquement mal fondées. Pourquoi alors, ceux qui sont passés - par la logique institutionnelle des recrutements - de l'autre côté de la barrière ne pourraient-ils 
pas être soumis à semblable critique et correction, et pourquoi ne pourrions-nous pas leur (nous) appliquer les mêmes jugements que ceux auxquels les apprentis doivent logiquement se soumettre pour payer leur droit d'entrée dans le métier? Pourquoi serions-nous normatifs pour les uns (étudiants) et relativistes pour les autres (pairs)?

Plutôt que de laisser filer le terme d'interprétation vers ses usages démagogiques, on peut essayer d'énoncer ce qui définit l'interprétation sociologique (au sens large du terme qui englobe l'ensemble des sciences des contextes sociaux) et de la distinguer de l'univers de l'herméneutique libre, c'est-à-dire des interprétations sauvages, incontrôlées, empiriquement non contraintes. Car l'enquête en sciences sociales est ponctuée d'actes d'interprétation (interprétation d'indices, de corrélations statistiques, de discours, de traces, d'opérations de sélection ou de codage, etc.) qui, lorsque le travail est bien fait, interviennent à tout moment de l'enquête.

\section{Les droits et devoirs de l'interprète}

5 Les interprétations (au sens de «thèses ») peuvent être qualifiées de scientifiques 1) si elles s'appuient sur des matériaux empiriques ; 2) si sont livrés, aussi précisément que possible, les principes théoriques de sélection puis les modes de production de ces matériaux; 3) si sont clairement désignés les contextes spatio-temporellement situés de la "mesure " (de l'observation); enfin, 4) si sont explicités les modes de fabrication des résultats à partir des matériaux produits (modes de traitement des données et, si possible, choix du type d' écriture scientifique).

6 Le travail interprétatif n'intervient donc pas après la bataille empirique, mais avant, pendant et après la production des «données » qui ne sont justement jamais données mais constituées comme telles par une série d'actes interprétatifs. Et l'on voit bien à quel point l'expression « interprétation du réel » est éloignée du métier réel de chercheur en sciences sociales dans la mesure où elle donne l'impression que celui-ci serait un « penseur » face au « réel », une sorte d'interprète final.

7 La qualité du travail d'enquête en sciences sociales réside d'abord et avant tout dans la finesse et la justesse des actes interprétatifs mis en œuvre à chaque moment de la construction de la recherche, de manière prospective mais aussi de façon rétrospective. Lorsque certains actes sont commis "à l'aveugle", leurs conséquences sur le travail doivent être mesurées par la suite pour comprendre ce qui a été fait sans le savoir dans le moment même de leur effectuation. La connaissance sociologique ne s'engendre et n'avance que par un incessant travail d'anticipation des actes de recherche à venir et de retour réflexif sur les actes antérieurs de recherche, à partir des acquis progressivement conquis grâce aux actes de recherche suivants. Les différents moments de la recherche ne sont donc jamais séparés comme le laisseraient supposer les schémas hypothéticodéductifs scolaires. De façon pragmatique, on pourrait ainsi énoncer que tout est bon, à n'importe quel moment du travail, pour mieux comprendre ce qui a été fait à n'importe quel autre moment.

8 Au lieu de polémiquer sans fin sur la valeur de tel ou tel concept, de tel ou tel paradigme, les chercheurs en sciences sociales gagneraient à livrer et à mettre en débat leurs actes aussi concrets qu'interprétatifs - de recherche, car c'est souvent dans les moments les plus anodins de l'enquête que les thèses les plus fortes sont posées sans être soutenues. Dans cette perspective, on passe de l'espace pseudo-démocratique des interprétations 
sauvages, délestées du poids de toutes contraintes empiriques d'énonciation, à l'espace des interprétations empiriquement contraintes et sociologiquement contrôlées par les anticipations et les retours réflexifs.

\section{Qu'est-ce qu'une surinterprétation ?}

9 On peut évidemment faire le départ entre les interprétations selon leur degré de solidité ou de force du point de vue du déploiement du raisonnement sociologique dans les divers moments de la recherche, et du point de vue du volume et de l'étendue du matériau interprété (« soutenir une thèse » fondée sur un seul entretien est toujours possible, mais si le chercheur développe un réseau articulé et cohérent de preuves fondé sur des questionnaires, des entretiens, de l'observation et de l'analyse de documents, sa thèse n'en aura que plus de force probatoire). Il y a aussi des interprétations moins pertinentes, moins adéquates que d'autres. Une partie spécifique des interprétations faibles, imprudentes ou inadéquates est constituée par ce que l'on pourrait appeler des surinterprétations.

Qu'est-ce qu'une surinterprétation? Est-ce que toute interprétation n'est pas, en un certain sens, surinterprétation? On pourrait le penser puisque les chercheurs en sciences sociales - y compris les plus « compréhensifs » d'entre eux - mettent généralement plus de sens dans les actions des enquêtés que ces derniers n'en mettent eux-mêmes lorsqu'ils agissent, ou même lorsque ces derniers commentent, à l'occasion, les actions effectuées. Mais on ne peut définir la surinterprétation comme surplus de sens par rapport aux significations que livrent les enquêtés à propos de ce qu'ils font, sentent, perçoivent, etc., car alors le risque serait grand d'avoir à rejeter comme mésinterprétations pour cause de surinterprétation toutes les interprétations qui n'agréeraient pas aux enquêtés. Si les enquêtés avaient scientifiquement le droit de rejeter certaines interprétations, alors il faudrait explicitement leur accorder un rôle dans la validation des thèses scientifiques ${ }^{1}$

11 Or les enquêtés jugent souvent, en lisant des comptes rendus de recherche qui portent sur leurs activités, leurs vies, etc., que les interprétations ne correspondent pas à ce qu'ils vivent, qu'elles déforment la réalité telle qu'ils la connaissent et la perçoivent. Ces mêmes enquêtés peuvent aussi estimer que les chercheurs exagèrent certains traits, certains comportements, certaines dimensions de l'activité sociale, etc., qui leur paraissent secondaires, annexes. Pire que cela, l'expérience montre que les enquêtés ne reconnaissent pas toujours ce qu'ils ont dit lorsqu'on leur présente la transcription la plus plate, sans commentaire, de leur discours oral («je n’ai pas dit ça», «je ne parle pas comme ça »...). Si ces réactions d'enquêtés aux produits de la recherche ne doivent pas laisser insensible le chercheur en sciences sociales (celui-ci devrait pouvoir les comprendre, à l'intérieur même de son modèle d'intelligibilité, comme des indicateurs de certains traits de son analyse et pourrait même trouver à cette occasion de quoi l'enrichir), il ne peut être question in fine de laisser aux enquêtés le soin de trancher entre les «bonnes » et les «mauvaises » interprétations, les interprétations «justes » et les interprétations «fausses », etc. ${ }^{2}$

En définitive, lorsque le sociologue fait correctement son travail, la signification des événements, des pratiques, des représentations, etc., qu'il propose constitue toujours un surplus, un rajout par rapport à ce qui se dit ou s'interprète déjà ordinairement dans le monde social. Interpréter c'est donc toujours surinterpréter par rapport aux interprétations (pratiques ou réflexives) ordinaires: choisir de filer une métaphore le 
plus loin possible, de privilégier une dimension des réalités sociales, une échelle particulière des contextes, etc., cela implique de rendre étranger à nos yeux comme aux yeux des enquêtés un monde ordinaire parfois tellement évident que nous ne le voyons plus vraiment.

Mais ce n'est évidemment pas de cette surinterprétation-là dont nous voulons parler. Nous distinguerons donc pour la clarté de notre propos trois types de surinterprétations :

- les surinterprétations dues aux décrochages interprétatifs par rapport aux situations interprétées (type 1) ;

- les surinterprétations produites par le décalage non objectivé, non contrôlé et non corrigé entre la situation du chercheur face aux matériaux étudiés et la situation des enquêtés (type 2) ;

- les surinterprétations engendrées par la combinaison de deux éléments : d'une part la surabondance des preuves apportées pour prouver la pertinence du modèle théorique utilisé, d'autre part la nature "littéraire " (par emploi de procédés d'écriture) de la production d'« effets de preuve » avec la profusion d'exemples " parfaits », qui s'opposent aux exemples et contre-exemples engendrés ordinairement par l'enquête empirique (type 3).

\section{Les décrochages interprétatifs : surinterprétations de type 1}

On assiste à un tel type de surinterprétation (type 1) lorsque les matériaux sur lesquels s'appuie l'auteur sont insuffisants (en richesse, en qualité...) pour soutenir les thèses qu'il propose. On a alors effectivement l'impression d'avoir affaire à un décrochage de l'interprétation par rapport aux situations interprétées. Cette sorte d'excroissance interprétative nous amène à estimer que l'auteur "en fait trop ", s'éloignant trop du matériau en sa possession.

Tout enseignant-chercheur trouverait de multiples exemples de versions malhabiles de ce type de surinterprétation qui témoignent de la difficulté à maîtriser les commentaires théoriques par rapport aux matériaux, mesures, indices sur lesquels ils portent. Ce genre de décrochage interprétatif vis-à-vis des données est très fréquent chez les apprentis chercheurs qui livrent, souvent sans parvenir à le contrôler (ou à le masquer), des interprétations trop lourdes pour le type ou le volume d'informations sur lesquels ils s'appuient. Certaines interprétations peuvent n'être fondées ainsi que sur un très court extrait d'interaction, un bref passage d'entretien ou un fragment de document. Comment décider si l'interaction verbale ou le discours de l'enquêté ne serait pas plutôt justiciable d'une autre interprétation, plus pertinente ? Rien dans le matériau présenté ne peut nous aider à aller plus loin, parce qu'aucune interprétation ne saurait reposer sur un seul extrait de matériau. Pour commencer à prendre, l'interprétation devra s'appuyer sur des exemples variés tirés d'interactions verbales récurrentes (montrant par exemple la réapparition fréquente d'un certain type d'attitude), ou sur une interaction verbale confirmée par des propos tenus lors d'un entretien, par le rapport que l'enquêté a pu entretenir avec la situation d'entretien et avec l'enquêteur, par des entretiens menés avec d'autres enquêtés, par des sources écrites, etc.

Se pose donc la question du degré de forçage de l'interprétation par rapport aux réalités évoquées. Les auteurs de mémoires de recherche utilisent souvent les schèmes 
interprétatifs comme des lits de Procuste, c'est-à-dire en y introduisant de force les faibles matériaux recueillis ${ }^{3}$. En matière d'étude de cas notamment ${ }^{4}$, plus l'interprétation repose sur des mesures empiriques multiples et théoriquement comparables et moins on court le risque de la surinterprétation. La multiplication des données susceptibles de servir au chercheur permet alors de " tisser serré » les différents fils de l'interprétation. Il faut tout simplement penser ici au gain interprétatif considérable qu'il y a à travailler, pour chaque petit point d'analyse, sur plusieurs informations qui viennent soit se confirmer mutuellement soit, au contraire, se contredire et permettre de raisonnablement mettre en doute la fiabilité d'une partie des informations possédées, l'interrogation sur ce défaut de fiabilité pouvant à son tour, si on la considère comme partie intégrante du travail interprétatif, permettre de relancer ou d'enrichir l'analyse d'ensemble.

La valeur relative des différentes interprétations ne dépend pas seulement (et peut-être même pas essentiellement) de leur qualité ou de leur force intrinsèque, mais de leur bon usage en fonction des données disponibles. Et c'est toute une science du kairos interprétatif, de l'occasion interprétative, un sens du dosage de ce qui peut être avancé à tel ou tel moment de l'analyse en fonction des matériaux soumis à interprétation qui est en jeu dans l'apprentissage du métier de chercheur en sciences sociales.

De son côté, la version habile-professionnelle (savante) se caractérise par une inflation verbaliste du discours interprétatif vis-à-vis des matériaux, c'est-à-dire par une surenchère ou un gonflement interprétatif sans conséquence (et notamment sans gain particulier) en matière de production de données empiriques ou de mode de traitement des données.

\section{La société de consommation}

19 L'ouvrage de Jean Baudrillard, La Société de consommation, est un exemple idéal-typique. Au contraire d'autres textes du même auteur écrits dans une veine encore plus essayiste ${ }^{5}$, il $s^{\prime}$ inscrit explicitement ${ }^{6}$ dans le champ des sciences sociales. Son sous-titre qui s'inspire directement du structuralisme ambiant de l'époque, ses références à des lieux, des objets, des phénomènes sociaux ou des situations sociales "réels» (le drugstore, le centre commercial Parly 2, le téléspectateur relaxé devant les images de la guerre du Viêt-nam, les informations télévisées ou radiophoniques concernant les morts sur les routes, la météorologie, la pollution, l'homme riche qui conduit sa $2 \mathrm{CV}$, la machine à laver...), l'usage de données chiffrées (taux de mortalité par CSP, consommation élargie des ménages...), tout cela contribue à ancrer l'ouvrage dans l'univers des textes de sciences sociales.

20 Toutefois, les exemples ne constituent pas un corpus dont on connaittrait les principes théoriques de sélection. L'auteur illustre ses interprétations par des exemples fabriqués, fictifs, par des «clichés » tirés de l'«actualité » (au sens large du terme), mais il n'est aucunement question d'enregistrements de faits empiriquement attestés (datés, localisés). Ces évocations de la réalité (vs construction méthodique et théoriquement contrôlée d'un corpus) ont pour seule fonction de produire des effets de réalité. On ne trouve donc pas véritablement dans l'ouvrage de preuves empiriques, mais des informations sur le monde social qui composent comme un décor de théâtre. Autrement dit, l'interprétation de la société de consommation par l'auteur ne se fonde pas sur des matériaux empiriques mais utilise des évocations empiriques non construites pour 
illustrer un propos construit en dehors de tout esprit et de toute pratique de l'enquête. Du fait de l'absence d'ancrage empirique, la lecture de l'ouvrage donne au lecteur animé par ce même esprit d'enquête l'impression de tableaux de pensée un peu aériens et ne touchant jamais vraiment terre, à l'image de ces nappes de brouillard planant sur la surface des étangs.

Ce type de majoration de l'expression verbale signifie bien souvent que l'auteur se contente de traduire dans un langage savant, philosophique, esthétique ou poétique, des thèmes ordinaires de l'air du temps (journalistique, publicitaire, politique, philosophique...), compensant le manque - ou la désespérante absence - de données empiriques par un savoir-faire essayiste. Il peut alors séduire les acteurs dont il flatte les thèmes de prédilection ${ }^{7}$.

\section{Le Dieu-télévision}

Pour ne pas en rester à l'exemple caricatural qui pourrait donner l'impression, trop commode, de la marginalité de ce genre de production, nous pouvons aborder un autre cas de surinterprétation partielle, momentanée, qui ne structure pas l'ensemble d'un texte. Au contraire de l'exemple précédent, celui que nous allons commenter est constitué par un ouvrage animé par l'esprit d'enquête et par la réflexivité concernant la méthode d'observation employée. Il s'agit de l'ouvrage d'Olivier Schwartz, Le Monde privé des ouvriers ${ }^{8}$.

En certains passages, qui détonnent dans le style d'ensemble du texte, l'auteur succombe au décrochage interprétatif eu égard aux situations interprétées. Il s'agit des moments où il cherche à saisir le sens de certaines pratiques télévisuelles observables en milieux populaires, pratiques consistant notamment à laisser continuellement allumé le poste de télévision.

24 À chaque fois que la télévision est évoquée, le propos s'enfle verbalement, et le lecteur éprouve un sentiment de décrochage par rapport aux descriptions des scènes de la vie familiale autour du poste de télévision. L'auteur écrit, par exemple : «Je pense aussi, précisément, à ces télévisions qui, dans de multiples foyers, fonctionnent des journées entières, même si personne ne les regarde. Qu'un visiteur arrive, et on le fait asseoir, on lui sert à boire, on bavarde, la télévision continue de fonctionner sans que personne l'écoute, mais sans non plus que qui que ce soit songe à l'arrêter. Le poste continue de saturer l'espace de son flux d'images et de sons, et rien que par ce courant ininterrompu, par ce continuum visuel et sonore enveloppant, il remplit l'équivalent d'une fonction nourricière, garantissant l'alimentation permanente $d u$ corps en stimulants perceptifs ou hallucinogènes. La télévision est l'une des grandes divinités du foyer ouvrier moderne9." L'impression de saturation de l'espace par les images et les sons des émissions télévisées n'est-elle pas une impression de chercheur qui prête attention à ce qui constitue un événement pour lui mais qui peut être vécu par les habitants du foyer avec une attention distraite, oblique ou sur le mode de la consommation nonchalante et du "savoir en prendre et en laisser " que Richard Hoggart observait dans les classes populaires anglaises ${ }^{10}$ ? On peut aussi s'interroger sur le profit heuristique qu'il y a à évoquer, dans une subite poussée d'emphase herméneutique, la «fonction nourricière » de la télévision ou encore à utiliser la métaphore de la « divinité ».

25 Le même style interprétatif est employé beaucoup plus loin dans l'ouvrage, où l'on retrouve la même hypothèse concernant la «saturation de la perception " dans la 
réception des images par le corps, l'auteur ajoutant que le «flux ininterrompu » d'images " autorise au spectateur une position de pure jouissance passive ${ }^{11}$ ». Là encore, on peut se demander ce qui peut justifier l'étude de la réception d'images télévisées en termes de "jouissance passive», sachant que tout acte de réception culturelle est toujours, contrairement à ce que l'on imagine ordinairement du fait de la connotation passive du terme, une réception active ${ }^{12}$. Le thème de la passivité face aux images télévisées constituant un thème récurrent et ordinaire des polémiques culturelles journalistiques ou essayistes lancées par les « défenseurs de la littérature » contre la télévision, il s'agirait davantage de le prendre comme objet d'étude que de le thématiser dans un langage savant.

Puis l'on retrouve la référence au sacré, mais la télévision devient alors la divinité de l'ensemble des foyers et non plus des seuls foyers ouvriers : « Divinité essentielle du foyer moderne, la télévision symbolise de façon très nette la vertu afférente à ce lieu, qui est de soutenir et de sécuriser la demande par la multiplication de 1'“effet de présence"13.» Dans ces moments le lecteur éprouve le net sentiment d'une excroissance interprétative se développant sur un corps empirique chétif.

\section{La leçon d'écriture}

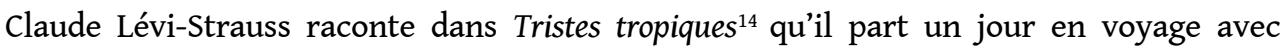
quelques Nambikwara, apportant avec lui des cadeaux qu'il compte distribuer à ses hôtes. Alors qu'ils sont arrivés au terme de leur voyage, il se passe « un incident extraordinaire » qui va déclencher l'imagination de l'anthropologue et lui faire écrire de longs développements sur l'écriture, ses usages et ses fonctions, le pouvoir et la connaissance, etc.

Lévi-Strauss raconte qu'il distribue des feuilles de papier et des crayons aux indigènes qui n'en font tout d'abord pas grand cas, mais qui les amènent tout de même un jour «à tracer sur le papier des lignes horizontales ondulées », cherchant «à faire de leur crayon le même usage " que lui. Mais, alors que généralement pour ceux qui s'y essayaient "l'effort s'arrêtait là », le « chef de bande voyait plus loin ». Avant même la description précise de l'«incident extraordinaire» annoncé plus haut, Lévi-Strauss nous livre d'emblée son interprétation à propos de ce qui s'est passé ce jour-là : le chef, à qui il prête une capacité à «voir plus loin» que les autres, aurait tout simplement «compris la fonction de l'écriture».

«Aussi m'a-t-il réclamé un bloc-notes et nous sommes pareillement équipés quand nous travaillons ensemble. Il ne me communique pas verbalement les informations que je lui demande, mais trace sur son papier des lignes sinueuses et me les présente, comme si je devais lire sa réponse. Lui-même est à moitié dupe de sa comédie; chaque fois que sa main achève une ligne, il l'examine anxieusement comme si la signification devait en jaillir, et la même désillusion se peint sur son visage. Mais il n'en convient pas; et il est tacitement entendu entre nous que son grimoire possède un sens que je feins de déchiffrer; le commentaire verbal suit presque aussitôt et me dispense de réclamer les éclaircissements nécessaires. Or, à peine avait-il rassemblé tout son monde qu'il tira d'une hotte un papier couvert de lignes tortillées qu'il fit semblant de lire et où il cherchait, avec une hésitation affectée, la liste des objets que je devais donner en retour des cadeaux offerts : à celui-ci, contre un arc et des flèches, un sabre d'abatis ! à tel autre, des perles ! pour ses colliers... Cette comédie se prolongea pendant deux heures. Qu'espérait-il ? Se tromper lui-même, peut-être ; mais plutôt étonner ses compagnons, les persuader 
que les marchandises passaient par son intermédiaire, qu'il avait obtenu l'alliance

du blanc et qu'il participait à ses secrets. » paragraphes, de «comédie » ou de «mystification » et qui avait contribué à créer « un climat irritant »- que Lévi-Strauss commence à interpréter l'événement dont il a été le témoin. D'emblée l'ethnologue déchiffre, dans le spectacle qui lui est donné à voir, l'usage de l'écriture «en vue d'une fin sociologique plutôt qu'intellectuelle » : «Il ne s'agissait pas de connaître, de retenir ou de comprendre, mais d'accroître le prestige et l'autorité d'un individu - ou d'une fonction - aux dépens d'autrui. Un indigène encore à l'âge de pierre avait deviné que le grand moyen de comprendre, à défaut de le comprendre, pouvait au moins servir à d'autres fins». Puis, à l'immédiate suite de ce bref commentaire, Lévi-Strauss esquisse les fondements d'une théorie générale des fonctions de l'écriture, glissant ainsi de la description et de l'interprétation ethnographique d'un moment de la vie des Nambikwara à des considérations beaucoup plus vastes - que nous ne commenterons pas en elles-mêmes ici - sur l'écriture.

Le scribe est "celui qui a prise sur les autres ». Rejetant l'hypothèse d'une fonction principalement et originellement «intellectuelle» (cognitive) ou "esthétique » de l'écriture, il rattache l'invention de l'écriture et le déploiement de ses usages à «la formation des cités et des empires, c'est-à-dire l'intégration dans un système politique d'un nombre considérable d'individus et leur hiérarchisation en castes et en classes ». L'écriture "paraît favoriser l'exploitation des hommes avant leur illumination", sa «fonction primaire» étant de «faciliter l'asservissement», d'" affermir les dominations ». Sautant du Pakistan oriental à l'Égypte, Sumer, la Chine, l'Afrique, l'Amérique précolombienne, pour terminer par l'exemple des États européens au XIX siècle, Lévi-Strauss voit même la "lutte contre l'analphabétisme " et l'« instruction obligatoire » (« qui va de pair avec l'extension du service militaire et la prolétarisation ») comme des éléments de « renforcement du contrôle des citoyens par le Pouvoir ».

Revenant sur l'élément déclencheur, Lévi-Strauss loue finalement la sagesse des «fortes têtes » qui vont se désolidariser de leur chef « après qu'il eut essayé de jouer la carte de la civilisation ». La sagesse tient au fait qu'ils « comprenaient confusément que l'écriture et la perfidie pénétraient chez eux de concert »; et Lévi-Strauss de rajouter : « Réfugiés dans une brousse plus lointaine, ils se sont ménagé un répit. » À la sagesse des " fortes têtes ", cependant, est tout de même opposé « le génie de leur chef » qui avait su percevoir « d'un seul coup le secours que l'écriture pouvait apporter à son pouvoir » et avait atteint ainsi «le fondement de l'institution sans en posséder l'usage ».

De fortes têtes néanmoins sages - figures des « sociétés authentiques » - qui sentent la perfidie liée à l'écriture en intuitant toute la force oppressive contenue en sa substance, un chef génial qui a pour sa part saisi en un clin d'œil le fondement de l'écriture, sa fonction sociale primaire d'asservissement, d'affirmation du pouvoir : Lévi-Strauss fait jouer aux acteurs (bons et perfides sauvages nambikwara) une scène qu'ils n'ont pas vécue. L'interprète sur-sollicite les "données" (la description d'une scène un peu extraordinaire de la vie quotidienne) et déborde généreusement les limites de ce qu'elles lui permettraient d'énoncer. Si les costumes et les décors appartiennent aux Nambikwara, le texte et la mise en scène sont de Claude Lévi-Strauss. Tout se passe comme si l'ethnologue profitait de cette scène pour énoncer une théorie de l'écriture qui n'est en rien fondée sur les données ethnographiques. La scène est davantage construite par l'auteur comme une parabole que comme une séquence de comportements observés mise 
en relation avec une série d'autres faits similaires ou différents ${ }^{15}$; elle est davantage l'occasion pour l'auteur de tirer une leçon sur l'écriture et le pouvoir que de faire la science exacte de ce qui se passa ce jour-là chez les Nambikwara. Concrètement, on n'a aucunement le sentiment que Lévi-Strauss interprète ici des produits de l'observation, mais qu'il prend prétexte d'une scène, qu'il constitue comme un événement (un « incident extraordinaire »), pour déployer une théorie générale et universelle de l'écriture conquise ailleurs, hors du travail d'enquête. Jacques Derrida, commentateur précis et prudent de cette leçon d'écriture, relevait dans De la grammatologie la surinterprétation opérée par l'ethnologue lorsqu'il écrivait : «L'écart le plus massif apparaîtra d'abord [...] entre le fait très mince de 1 '«incident extraordinaire » et la philosophie générale de l'écriture. La pointe de l'incident supporte en effet un énorme édifice théorique ${ }^{16}$. $»$ Et l'on pourrait rajouter que l'incident relaté s'effondre sous le poids du commentaire théorique qui ne trouve guère ainsi de soutien empirique ${ }^{17}$.

\section{Les décalages non contrôlés entre chercheurs et enquêtés : surinterprétations de type 2}

Les surinterprétations de type 2 se caractérisent par l'oubli dans l'interprétation des conditions réelles dans lesquelles les acteurs étaient amenés à agir, penser, voir, percevoir, etc., c'est-à-dire par l'oubli du décalage entre l'œil savant (les conditions savantes de perception du monde social) et l'œil ordinaire (les conditions ordinaires de perception du monde social liées aux formes de vie sociale). En pareil cas, le chercheur ignore la différence de situation et de perspective entre lui et ceux qu'il étudie et prête ainsi à ces derniers des capacités visuelles, auditives, sensitives, cognitives plus "grandes» que (ou simplement différentes de) celles qu'ils possèdent réellement. Il projette alors le rapport qu'il entretient avec l'objet de connaissance en tant que sujet connaissant dans la tête (les représentations, les structures de perception, etc.) de ceux qu'il étudie.

On trouve une telle réflexion épistémologique chez Pierre Bourdieu lorsque celui-ci met en garde contre l'intellectualisme qui consiste à «introduire dans l'objet le rapport intellectuel à l'objet ", c'est-à-dire à "substituer au rapport pratique à la pratique le rapport à l'objet qui est celui de l'observateur ${ }^{18}$ ». Mais toutes les situations de surinterprétation qui tiennent au décalage non contrôlé entre l'univers du chercheur et l'univers des enquêtés ne concernent pas exclusivement la différence entre action et connaissance, sens pratique et réflexivité, rapport pratique à la pratique et vision théorique de la pratique, temps de l'urgence et temps intemporel de la science, etc.

Par exemple, lorsque Paul Veyne évoque les bas-reliefs représentant les différents épisodes des campagnes de Dacie et ornant en une frise spirale le tour de la colonne Trajane élevée à Rome en l'honneur de l'empereur romain Trajan (98-117), vainqueur des Daces en $112^{19}$, il relève la très faible pertinence des interprétations de ces scènes en termes d'art de propagande impérial dans la mesure où ces images étaient architecturalement, spatialement, invisibles par le public de l'époque. Visibles, descriptibles et analysables, ces images ne le sont que pour le savant qui a les moyens de travailler sur la reproduction de ces scènes (une bande d'environ quinze mètres de long), hors contexte spatial d'origine, et de les voir comme personne avant lui n'a pu les voir ${ }^{20}$. 
En invoquant l'art de propagande, l'interprète «en fait trop» et, finalement, manque l'interprétation.

De même, combien de surinterprétations dans les exégèses contemporaines des textes philosophiques grecs ignorantes du fait que les Grecs «écrivaient en scriptio continua, c'est-à-dire sans intervalles entre les mots, ce qui - l'expérience le montre - rend la lecture à haute voix pratiquement nécessaire ${ }^{21} »$. Dans un remarquable texte d'anthropologie historique, Jesper Svenbro met en évidence le fait que, pour les anciens grecs, le texte écrit apparait incomplet sans la voix qui l'oralise. Et, au moment de la lecture, la voix n'appartient plus au lecteur mais à l'écrit, car la lecture fait partie intégrante du texte. Dans une telle économie des représentations, la voix se met au service du texte et celui qui prête sa voix est dominé : «Écrire, c'est être dominant, actif, victorieux - à condition de trouver un lecteur prêt à céder. Lire, si l'on se décide à le faire (car le lecteur - s'il n'est pas de condition servile - est évidemment libre de refuser de lire), c'est se soumettre à la trace écrite du scripteur, c'est être dominé, c'est occuper la position du vaincu. C'est se soumettre à cet éraste métaphorique qu'est le scripteur. S'il est honorable d'écrire, il n'est pas sûr que la lecture, elle, soit sans problèmes, étant vécue comme une servitude et comme une "passivité" (est "passif" celui qui subit l'écriture). [...] Et on peut donc penser que la lecture, tâche que les Grecs laissent volontiers aux esclaves, comme dans le Théétète de Platon, doit se pratiquer avec modération pour ne pas devenir un vice. Ou mieux : celui qui lit ne doit pas s'identifier au rôle du lecteur s'il veut rester libre, c'est-à-dire libre des contraintes imposées par l'Autre. Mieux vaut rester tà grammata phaûlos, "faible en lecture", à savoir capable de lire, mais sans plus ${ }^{22}$.» Il est évident que les conditions de réception d'un texte (et, partant, les conditions de travail sur ce texte) sont différentes selon que le texte est lu oralement ou silencieusement, avec les yeux; selon aussi le sens que prend l'acte de lire pour celui qui l'effectue. Nous pouvons donc, aujourd'hui, sans le savoir, faire parler les textes grecs non seulement à travers d'autres intérêts culturels que ceux des philosophes grecs, mais aussi, et cela est beaucoup moins contrôlé, à travers le prisme d'autres représentations de l'acte de lire et d'autres techniques intellectuelles de travail sur les textes. Les exégètes peuvent ainsi oublier dans leurs commentaires l'écart entre les modes savants-contemporains de traitement des textes et les modes de traitements des textes propres aux lecteurs de l'époque.

37 C'est toujours le même type d'erreur qui amène à traiter et à commenter « littérairement » ce qui ne constitue nullement de la littérature. Par exemple, les poèmes oraux en Grèce ancienne ne constituent en aucun cas un genre littéraire. Énoncés oralement dans des contextes rituels ou cérémoniels particuliers, orientés vers des fonctions sociales pratiques (paroles de banquets invitant aux libations et à l'amour...), ils n'entrent absolument pas dans le même circuit de production du sens que le texte littéraire écrit, destiné à un lecteur plutôt qu'à un auditoire, lu solitairement en silence et rendant possible la mise en œuvre d'un mode herméneutique d'appropriation du texte ${ }^{23}$. Faire la sémiologie des mythes ou l'étude littéraire des odes grecques alors que mythes et odes sont justiciables d'une analyse plus pragmatique, contextuelle, relève de la faute de surinterprétation par oubli des conditions concrètes d'existence des réalités mythicorituelles ou des poésies chantées ${ }^{24}$. De plus, en plaquant ses catégories littéraires, scientifiques ou philosophiques d'analyse sur une telle situation culturelle, le chercheur contemporain aplatit toutes les formes d'expression et de circulation de la culture de l'époque en prenant objectivement parti pour ceux qui, au cœur même de l'événement, 
vont historiquement dans le sens du travail d'invention de la littérature, de la science ou de la philosophie. La surinterprétation littéraire, philosophique ou scientifique des produits culturels de l'époque manque par là même une partie importante de l'objet étudié.

Dans de telles conditions, les commentaires qui mettent entre parenthèses les conditions effectives de circulation, de transmission et d'appropriation des textes philosophiques, des mythes ou des poésies, sont bien en régime de surinterprétation (type 2). Au lieu d'intégrer dans leur travail interprétatif les conditions réelles dans lesquelles les acteurs étaient amenés à agir, sentir, penser, les chercheurs font subir aux produits culturels étudiés un transfert scientifiquement illégal ${ }^{25}$. Le chercheur est alors plus proche de la démarche artistique (créative) que de la démarche sociologique : il dit du mythe, de la poésie lyrique ou du texte philosophique ce que peut en dire et en faire celui qui dispose de techniques intellectuelles scripturales et graphiques contemporaines, mais pas - et c'est pourtant le but de l'étude sociologique, anthropologique ou historienne - ce qu'ils sont (leur mode d'énonciation, de transmission, etc.) pour les acteurs de l'époque.

On pourrait, à propos de tels exemples, parler d'anachronisme, puisqu'il s'agit bien de cela, mais on ne décrirait pas suffisamment précisément le problème en le désignant ainsi. Il s'agit en effet d'un anachronisme spécifique lié à l'oubli des conditions les plus matérielles d'action, de représentation, de réception des œuvres culturelles dans lesquelles se trouvaient insérés les protagonistes de l'époque considérée. Pour éviter la surinterprétation en ce domaine, il faut donc prêter une attention particulière à la matérialité des objets, des actes, des gestes, des situations. Cela nous ramène à l'impératif de la description fine de réalités souvent simplement évoquées ou survolées par les travaux en sciences sociales.

Si l'historien fait de la surinterprétation à propos de la colonne Trajane en commentant des images comme si elles pouvaient être vues alors que leur taille et leur disposition spatiale les rendent invisibles aux yeux des acteurs ordinaires, s'il peut être encore surherméneute lorsqu'il lit les mythes, les poésies ou les textes philosophiques grecs à partir d'autres conventions, d'autres techniques intellectuelles, d'autres procédures que celles qu'étaient en mesure de mettre en œuvre les lecteurs ou les énonciateurs de l'époque, on peut penser aussi au cas des spécialistes de l'art qui prêtent aux visiteurs cultivés des musées (pressés ou attentifs, passionnés ou nonchalants...) des compétences en histoire de l'art, ou au cas des sémiologues qui confèrent aux spectateurs (distraits ou absorbés...) et téléspectateurs (affairés à la cuisine ou captivés par le film...) une culture et une disposition sémiologique semblables à celles qu'ils mettent en œuvre dans leur analyse des émissions télévisées ou des spectacles culturels. Or, aussi bien les compétences effectivement possédées par le public (qui sont socialement inégalement distribuées mais qui, tout particulièrement en ces domaines, restent l'apanage d'une très faible minorité) que les conditions effectives de réception du message (par exemple, l'analyse sémiologique ne peut se faire que dans le temps long de la science alors que le téléspectateur ordinaire regarde la télévision dans le temps réel du déroulement des images) rendent la plupart du temps impossible la vision sémiologique spontanée ou l'interprétation d'une œuvre picturale armée de connaissances en histoire de l'art. La remarque est encore plus pertinente à propos de l'art urbain qui n'est plus vu comme tel ( i.e. dans sa dimension esthétique) par ceux pour qui il constitue un décor urbain ordinaire. 
41 Les chercheurs dotés d'une grande connaissance savante, érudite des œuvres (picturales, textuelles, architecturales, etc.) ne peuvent bien sûr qu'être déçus par les réceptions réelles (les réceptions telles qu'elles se font dans telle communauté, à tel moment et dans telles conditions matérielles), nécessairement moins fouillées, moins riches, mais aussi, bien souvent, prises dans les contresens historiques, les anachronismes, les bricolages interprétatifs ${ }^{26}$. Ils ne peuvent de même qu'être désappointés par l'absence d'intérêt pour des éléments jugés centraux de l'œuvre et par le goût appuyé des profanes pour des traits habituellement supposés annexes, secondaires par les spécialistes.

\section{La surabondance d'exemples parfaits : surinterprétations de type 3}

42 L'exemple qui suit est-il encore un cas de surinterprétation? On pourrait en douter. L'auteur, dont l'esprit d'enquête a animé une grande partie de l'œuvre, ne sur-sollicite pas ses données en déboîtant ainsi le commentaire de son support empirique (surinterprétation de type 1); il ne pèche pas davantage par oubli des conditions effectives, concrètes dans lesquelles se meuvent les enquêtés (surinterprétation de type 2), mais tisse au contraire un réseau serré de preuves qui font la force indéniable de l'interprétation proposée. Le problème se situe ici presque à l'opposé de celui que pose la surinterprétation de type 1 : dans le premier cas on déplore l'absence de «données » ou les licences herméneutiques que s'accordent les auteurs eu égard au volume, à la qualité ou à la nature des matériaux empiriques mobilisés; dans ce dernier cas on peut en revanche s'interroger d'une part sur la surproduction de procédés déployés pour « faire preuve ", c'est-à-dire pour prouver la pertinence du modèle théorique utilisé, et d'autre part sur la nature (ou la logique) même de ces procédés.

À force de consolider, voire de fortifier sa théorie, le sociologue peut progressivement inverser le cours scientifiquement normal des choses. Il bascule de la volonté de comprendre et d'expliquer les faits sociaux qui expose fatalement la théorie à de multiples transformations, adaptations et, dans certains cas, à de radicales remises en question, au désir, conscient ou inconscient, de gérer le patrimoine conceptuel qui amène progressivement à éviter de " voir » ce qui pourrait faire contre-exemple, ce qui pourrait entrer en contradiction avec la belle mécanique théorique. En l'état actuel des choses, l'espace scientifique concurrentiel conduit, qu'on le veuille ou non, vers la défense de sa théorie ${ }^{27}$ et cette défense peut entrainer à son tour vers une logique de l'enfermement théorique et de la dénégation des faits. Cette logique qui, à terme, mène droit dans le mur du dogmatisme interprétatif doit par conséquent être sérieusement contrôlée et contrariée par la logique de l'enquête et de la confrontation avec la diversité des « faits ».

Le cas qui nous préoccupe ici, La Distinction de Pierre Bourdieu, est donc un cas limite choisi comme tel - de surinterprétation par surabondance de «preuves» contribuant à valider le modèle, l'auteur tendant sans cesse à « en rajouter » et à faire ainsi défiler à la barre les éléments - et seulement ceux-là - témoignant en faveur de la pertinence interprétative du modèle proposé. 


\section{Entre catégories savantes et catégories ordinaires} restent des catégories à expliquer) et des catégories savantes, on finit par se demander si la preuve de la pertinence de l'interprétation sociologique n'est pas finalement produite par les incessants glissements sémantiques. De nombreux passages de l'ouvrage ressemblent presque trop à nos mythologies (littéraires et cinématographiques) sur les mondes populaires, petits-bourgeois et bourgeois. L'auteur parait parfois en rajouter dans l'écriture qui, pour évoquer le monde populaire, peut adopter un accent rabelaisien («Et la philosophie pratique du corps masculin comme une sorte de puissance, grande, forte, aux besoins énormes, impérieux et brutaux, qui s'affirme dans toute la manière masculine de tenir le $\operatorname{corps}^{29}$ »; « la viande, nourriture par excellence forte, donnant de la force, de la vigueur, du sang, de la santé, est le plat des hommes ${ }^{30}$ ", etc.). On assiste alors à un gonflement stylistique.

\section{Des exemples sur mesure}

L'alliage des catégories savantes et ordinaires est notamment à l'œuvre dans les moments

- très fréquents - où l'auteur se situe entre la description phénoménologique et l'analyse sociologique de comportements (gestuels et langagiers) socialement marqués. Se pose dès lors la question du statut des exemples donnés, des scènes simplement évoquées ou soigneusement décrites. Dans la grande majorité des cas, Bourdieu décrit des scènes 
observées avec grande acuité, mais qui ne sont pas tirées d'un travail d'observation systématique des comportements. Elles ne font pas partie d'un corpus théoriquement et méthodologiquement construit (ce qui supposerait de dire comment les observations ont été effectuées, à partir de quelle construction de l'objet, quelle place les scènes rapportées occupent dans la nécessaire dispersion-variation des situations observées, etc.) mais relèvent de ce que l'on pourrait appeler l'exemple sur mesure. Ces scènes sont donc écrites pour exemplifier le schéma théorique (les oppositions substance/forme ; matériel/ symbolique ; nécessité/liberté...).

Parfois, l'exemple imaginaire (mais qui pourrait avoir été réellement observé) est clairement énoncé pour faire immédiatement comprendre - pédagogiquement - le sens d'une proposition théorique. C'est le cas ainsi de l'évocation d'« un vieil artisan » pour exemplifier le concept de "formule génératrice ${ }^{31}$ ». Impossible de nier l'existence de situations analogues à celle donnée en exemple par l'auteur (seule la mauvaise foi théorique amènerait le chercheur à nier en avoir déjà rencontrées au cours de ses enquêtes), mais le cas sur mesure, qui vient témoigner en faveur du schème théorique, peut faire oublier qu'il est possible de montrer par la recherche empirique que toutes les situations sociales ne relèvent pas de ce modèle ${ }^{32}$.

Mais le plus souvent, les descriptions phénoménologiques des manières de faire ou de parler ne sont ni des exemples purement imaginaires, ni des comptes rendus de situations singulières tirées d'une série raisonnée d'observations. Elles ont un air de parenté avec des scènes littéraires, sociologiquement probables, telles qu'on en trouve, par exemple, dans les romans de Flaubert ${ }^{33}$. Le sociologue a alors toute latitude - et peu de contraintes empiriques - pour décrire des scènes qui mobilisent les oppositions théoriques placées au cœur de l'interprétation sociologique :

« [...] dans les situations ordinaires de l'existence bourgeoise, les banalités sur l'art, la littérature ou le cinéma ont la voix grave et bien posée, la diction lente et désinvolte, le sourire distant ou assuré, le geste mesuré, le costume de bonne coupe et le salon bourgeois de celui qui les prononce [...]».

«En matière de langage, c'est l'opposition entre le franc-parler populaire et le langage hautement censuré de la bourgeoisie, entre la recherche expressionniste du pittoresque ou de l'effet et le parti de retenue et de feinte simplicité (litotès en grec). Même économie de moyens dans l'usage du langage corporel: là encore, la gesticulation et la presse, les mines et les mimiques, s'opposent à la lenteur - "les gestes lents, le regard lent" de la noblesse selon Nietzsche -, à la retenue et à l'impassibilité par où se marque la hauteur."

«Il serait facile de montrer par exemple que les Kleenex, qui demandent qu'on prenne son nez délicatement, sans trop appuyer et qu'on se mouche en quelque sorte du bout du nez, par petits coups, sont au grand mouchoir de tissu, dans lequel on souffle très fort d'un coup et à grand bruit, en plissant les yeux dans l'effort et en tenant le nez à pleins doigts, ce que le rire retenu dans ses manifestations visibles et sonores est au rire à gorge déployée, que l'on pousse avec tout le corps, en plissant le nez, en ouvrant grande la bouche, en prenant son souffle très profond ("j'étais plié en deux"), comme pour amplifier au maximum une expérience qui ne souffre pas d'être contenue et d'abord parce qu'elle doit être partagée, donc clairement manifestée à l'intention des autres ${ }^{34}$. »

51 Mais le talent d'écriture de Bourdieu le fait passer parfois de descriptions désingularisées (non situées dans le temps et dans l'espace géographique et social), qui pourraient être comme le résumé idéal-typique d'une multitude d'observations effectuées ${ }^{35}$ - c'est le cas de la fréquentation du café en milieux populaires ${ }^{36}$ - à la description de scènes singulières qui relèvent, là encore, davantage de la scène littéraire que du compte rendu d'enquête 
circonstancié. Dans ces cas, on remarque que l'auteur atteint un degré de détail tel qu'il ne peut convenir qu'à la description d'une scène particulière, singulière, mettant en scène des personnages singuliers. Or ces personnages sont fictifs du point de vue sociologique. Il y a donc bien une volonté stylistique délibérée de produire un «effet littéraire ", qui n'est autre qu'un " effet de réel ${ }^{37}$ ", et l'on peut alors se demander si les effets de réel littéraires ne prennent pas le pas sur les effets de connaissance sociologiques dans ces descriptions de situations singulières visant à exemplifier un style de vie, un habitus ou une vision du monde ${ }^{38}$ :

« On tend à ignorer le souci de l'ordonnance stricte du repas : tout peut ainsi être mis sur la table à peu près en même temps (ce qui a aussi pour vertu d'économiser des pas), en sorte que les femmes peuvent en être déjà au dessert, avec les enfants, qui emportent leur assiette devant la télévision, pendant que les hommes finissent le plat principal ou que le "garçon", arrivé en retard, avale sa soupe. [...] On peut ainsi, au café, se contenter d'une cuillère à café que l'on passe au voisin, après l'avoir secouée, pour qu'il "tourne son sucre" à son tour. [...] De même on ne change pas les assiettes entre les plats. L'assiette à soupe, que l'on nettoie avec le pain, peut ainsi servir jusqu'à la fin du repas. La maîtresse de maison ne manque pas de proposer de "changer les assiettes", en repoussant déjà sa chaise d'une main et en tendant l'autre vers l'assiette de son voisin, mais tout le monde se récrie ("ça se mélange dans le ventre") et si elle insistait, elle aurait l'air de vouloir exhiber sa vaisselle ${ }^{39}$."

Encadrant (ou encadrés par, comme on voudra) les commentaires des données issues des enquêtes quantitatives, les évocations littéraires, situées dans le registre de la description de comportements singuliers de personnages singuliers, ont tendance à capturer les classes ou les fractions de classe (populaires, petites bourgeoises ou bourgeoises), à les enfermer dans les cas singuliers. C'est la classe ou la fraction de classe qui se donne à voir dans la singularité du cas : les exemples livrent, par synecdoque, comme la quintessence d'un style de vie ou d'un habitus de classe.

\section{Le statut ambigu des photographies}

De nombreuses photographies émaillent l'ouvrage de Pierre Bourdieu. Or, ces photographies fonctionnent comme les petites descriptions phénoménologico-littéraires qui singularisent et exemplifient le propos théorique, mais avec un problème supplémentaire, à savoir que nombre d'entre elles ${ }^{40}$ ne sont pas commentées par l'auteur et qu'elles sont dès lors à la fois sur-signifiantes (elles paraissent montrer ce que l'auteur décrit par ailleurs : des manières de se tenir plus ou moins droit ou relâché, des distances spatiales plus ou moins grandes entre interlocuteurs, des tables plus ou moins garnies de victuailles et de plats, et plus ou moins strictement ordonnées...) et sous-signifiantes (les images ne nous disent rien ${ }^{41}$ et, lorsqu'elles semblent " parler d'elles-mêmes ", il faut tout particulièrement nous en méfier car le risque est grand alors de projeter nos petites mythologies sociales personnelles ou collectives).

De même que pour les scènes de description, on s'attendrait à ce que l'auteur nous dise dans quels contextes, à quelles occasions, etc., les photographies ont été prises, ce qui pourrait souvent contribuer à expliquer les différences visibles : repas familial ou repas avec invités, repas du dimanche ou repas en semaine, photographie officielle et publique pour un journal ou photographie au statut plus incertain lorsqu'elle est prise par le sociologue à la suite d'un entretien, etc. Pour faire véritablement corpus et pas seulement "bon-exemple-pour-la-théorie ", les photographies devraient ainsi être commentées, de même que les conditions de prise de vue. Ne sont présentées ici que les photographies les 
plus «parlantes", celles qui viennent témoigner en faveur de la thèse centrale de l'auteur. Du même coup, leur présence participe, là encore, davantage d'un effet de réel que d'un réel effet de connaissance. La production d'un effet de connaissance sociologique supposerait l'analyse d'un corpus de photographies prises dans des conditions relativement similaires, dans des familles socialement variées et clairement situées (sous l'angle des capitaux économique et culturel notamment).

\section{La lecture des données statistiques}

Enfin, lus de manière comparative, les différents moments du texte laissent apparaître parfois des surinterprétations de type 1. Par exemple, à la suite d'un passage consacré au "bon vivant ${ }^{42}$ ", on peut lire que $64 \%$ des cadres supérieurs, professions libérales et industriels jugent que «le Français mange trop » contre seulement $46 \%$ des ouvriers. Or, si l'écart statistique est amplement significatif pour susciter le commentaire qui le précède, on peut toutefois se demander si les $46 \%$ d'ouvriers qui portent le même jugement que les classes supérieures ne devraient pas être l'objet d'une analyse sociologique spécifique, analyse qui forcerait notamment l'auteur à distinguer les fractions de classe au sein des milieux populaires aussi systématiquement qu'il le fait pour les autres classes. En tout état de cause, les $46 \%$ se laissent difficilement enfermer dans la description phénoménologique qui précède le commentaire des chiffres.

De même, à la suite de l'extrait suivant :

"Cette manière d'introduire la rigueur de la règle jusque dans le quotidien (on se rase et on s'habille chaque jour dès le matin, et pas seulement pour "sortir"), d'exclure la coupure entre le chez soi et le dehors, le quotidien et l'extra-quotidien (associé, pour les classes populaires, au fait de s'endimancher) ne s'explique pas seulement par la présence au sein du monde familial et familier de ces étrangers que sont les domestiques et les invités. Elle est l'expression d'un habitus d'ordre, de tenue et de retenue qui ne saurait être abdiqué ${ }^{43}$. »

57 Si l'on se rapporte au tableau 19 rassemblant les résultats statistiques concernant les "Variations des manières de recevoir ", on constate encore une fois que si les écarts sont significatifs entre ouvriers et cadres supérieurs, industriels et professions libérales lorsqu'on leur demande s'ils aiment que leurs invités soient en tenue décontractée (79,7 \% pour les premiers et $58,6 \%$ pour les seconds), on peut tout aussi bien remarquer que la préférence des classes supérieures va vers cette modalité de la réception et que seuls $30,6 \%$ d'entre eux souhaitent que leurs invités soient élégants. Ce que l'on peut contester ici, ce n'est pas le commentaire tel qu'il est fait des écarts entre groupes ou classes, mais l'élision de tout ce qui pourrait faire contre-exemple, de tout ce qui pourrait jeter le doute ou apporter quelques nuances à l'architecture théorique.

\section{Les interprétations et leurs limites de pertinence}

En pratique, toutes les interprétations ne se valent pas. Mais si toutes les interprétations ne sont pas équivalentes, leur valeur n'est toutefois jamais fixée et acquise une fois pour toutes. Et cette variabilité des valeurs ou des pertinences interprétatives est essentiellement due au fait que ce qui définit sociologiquement la pertinence d'une interprétation, c'est sa capacité à rendre raison du monde social et non sa force intrinsèque, sa rigueur logique ou sa finesse argumentative. Quelle que soit la pertinence attestée, éprouvée dans l'enquête empirique, d'une interprétation complexe, 
relativement cohérente et conceptualisée (une théorie du social), aucun chercheur en sciences sociales ne sera jamais dispensé de faire la preuve empirique sur d'autres terrains, d'autres objets, d'autres époques, d'autres populations, etc., de cette pertinence. Une interprétation (un ensemble cohérent de schèmes interprétatifs discrets aussi bien qu'une grille d'interprétation conceptualisée, formalisée) a donc toujours des limites de validité, un champ toujours limité de pertinence.

Puisqu'il a été longuement question dans les pages précédentes de surinterprétations, on pourrait faire remarquer qu'une grande partie des travaux en sciences sociales souffre davantage de sous-interprétation: analyses poussives, sociographies et autres descriptions plates nous livrent un travail interprétatif embryonnaire. Ce constat nous amène à formuler la proposition selon laquelle toute interprétation sociologique pertinente est une surinterprétation contrôlée. Aucune règle de méthode ne permettrait de trouver à coup sûr le «bon niveau » d'interprétation (ni trop haut - surinterprétation - ni trop bas sous-interprétation). Toute interprétation, pour ne pas en rester à ces plats commentaires de tableaux ou à ces récits et à ces descriptions qui laissent le lecteur en quête d'explications sur sa faim, est potentiellement une surinterprétation dans la mesure où elle prend des risques. Et les risques de surinterprétation sont limités lorsque le travail interprétatif est soigneusement contrôlé par les données, par la réflexion sur leurs conditions de production, par la comparaison de ces données avec d'autres séries de données produites par d'autres, dans d'autres conditions, etc. C'est là que se fait la différence entre le dérapage contrôlé volontaire du professionnel qui tient la route de l'interprétation (respectant les données et les contraintes qu'elles font toujours - par leur diversité, leur apport de contre-exemples ou leur incohérence relative - inévitablement peser sur le discours du chercheur) et le dérapage incontrôlé de l'amateur - ou du professionnel en relâche - qui mène droit dans le décor de la surinterprétation.

Les concepts sociologiques (au sens large du terme) sont des mots qui n'ont aucune force ou aucune valeur scientifique en soi (i.e. purement théorique), mais qui ne valent que dans leur rencontre, leur confrontation avec le monde social et, finalement, dans leur capacité à capter et à organiser des éléments du monde social. La manière par conséquent dont nous avons abordé les problèmes posés par les surinterprétations en sciences sociales est fidèle à cette perspective : il n'existe pas de surinterprétation sociologique que l'on pourrait débusquer du point de vue strictement linguistique, logique ou étroitement argumentatif, mais des surinterprétations repérables au niveau des rapports que l'interprétation entretient avec les situations interprétées.

61 Enfin, pour cadrer le sens de ce texte et éviter les malentendus concernant son statut, il n'est pas inutile de rappeler que les réflexions épistémologiques qui viennent d'être formulées ici sont des réflexions de chercheur au travail pour qui le mot « enquête » n'est pas qu'une simple invocation verbaliste. En effet, pèse aujourd'hui sur ceux qui réfléchissent sur leur discipline, leurs savoirs, leurs méthodes et leurs mises à l'épreuve des faits un soupçon d'inutilité, de débauche luxueuse de temps qui marquerait une absence de travail d'enquête ou l'ennui du travail « de terrain ». Certains disqualifient par avance toute réflexion épistémologique comme réflexion futile, stérile, prétentieuse ou verbeuse. Et c'est évidemment toujours ceux qui ont un intérêt tout particulier au maintien de l'ordre scientifique en son état et dont l'épistémologie " va de soi ", « va sans dire ", qui n'ont aucun intérêt à voir advenir de nouvelles réflexions. La disqualification est donc plus difficile et, en tout cas, est forcée de se révéler sous son vrai jour et de dévoiler son moteur, lorsque ceux qui entreprennent de réfléchir n'ont pas quitté le 
chemin de l'enquête et y retournent d'autant plus volontiers que leurs réflexions épistémologiques collectives améliorent la qualité de leurs travaux empiriques et amplifient leur imagination sociologique en matière de construction des objets. L'épistémologie, lorsqu'elle est leçon tirée du travail de recherche et invitation à retourner sur le métier, n'a rien d'un préalable incontournable et un peu terroriste à l'enquête qui empêcherait l'enquête elle-même par peur de la faute commise. Guide, aide, appui ou coup de main, mais jamais droit de passage ou préalable.

\section{NOTES}

1. C'est ce que fait F. Dubet dans sa Sociologie de l'expérience, Paris, Seuil, 1994. Les sociologues doivent soumettre aux groupes d'acteurs avec lesquels ils travaillent dans le cadre d'une intervention sociologique leurs « interprétations sociologiques ». Puis les acteurs " sont invités à interpréter à leur tour les analyses des sociologues, à y réagir ». Dans un tel cadre de validation des interprétations sociologiques, le chercheur peut juger "fausse " son analyse si elle a été rejetée comme non "vraisemblable aux yeux de ceux qui [sont] le mieux armés pour en discuter». F. Dubet insiste donc sur la double destination de l'argumentation sociologique : «la communauté scientifique, avec ses critères propres, et les acteurs, qui maîtrisent d'autres données». Dans l'«espace d'argumentations réciproques» entre sociologue et acteurs, «le sociologue peut trouver matière à construire ses raisonnements et ses hypothèses; il peut aussi y fonder certaines formes de validation » (p. 244-252).

2. Si l'on décidait de la qualité ou de la pertinence de l'interprétation en fonction du point de vue des enquêtés, peu de travaux de sociologie de l'art résisteraient à la critique des artistes ou des critiques artistiques.

3. Cf. M. Weber, Essais sur la théorie de la science, Paris, Presses Pocket, Agora, 1992, p. 178.

4. Ce qui est le plus fréquent pour les apprentis qui travaillent le plus souvent sur le mode artisanal.

5. Par exemple À l'ombre des majorités silencieuses ou la fin du social, Paris, À l'imprimerie quotidienne, Cahier d'utopie quatre, 1978.

6. L'un de ses préfaciers juge que le livre «est une contribution magistrale à la sociologie contemporaine " et se risque à affirmer qu' " il a certainement sa place dans la lignée des livres comme De la division du travail social de Durkheim, La Théorie de la classe de loisir de Veblen ou La Foule solitaire de David Riesman ». Cf. J.-P. Mayer, "Avant-propos », in La Société de consommation, ses mythes, ses structures [1970], Paris, Gallimard, 1985, p. 13.

7. Ce que nous visons ici, ce ne sont pas les «essais" en tant que tels, mais le style essayiste lorsqu'il est à l'œuvre à l'intérieur du champ des sciences sociales. C'est la confusion des genres ou l'importation non contrôlée de certains genres dans le monde des sciences sociales, en vue notamment de s'épargner les affres de l'enquête empirique, qui posent problème et non l'existence de genres différenciés. Notre jugement a le champ des sciences sociales comme limite de validité et ne constitue pas une attaque contre le genre " essai » en général. On pourrait dire de même des sociologues-poètes, des sociologues-métaphysiciens, des sociologues-journalistes, des sociologues-idéologues, etc.

8. O. Schwartz, Le Monde privé des ouvriers. Hommes et femmes du Nord, Paris, Presses universitaires de France, 1990. Précisons que les remarques critiques que nous émettons sur ce texte n'ont pas 
pour principe le (malin) plaisir d'épingler un auteur en flagrant délit de surinterprétation, mais le souci de faire partager à cet auteur nos interrogations sur des moments (que nous jugeons) problématiques de l'interprétation.

9. O. Schwartz, ibid., p. 94-95. C'est nous qui soulignons.

10. R. Hoggart, La Culture du pauvre. Étude sur le style de vie des classes populaires en Angleterre, Paris, Ed. de Minuit, 1970.

11. O. Schwartz, op. cit., p. 382. Il parle plus loin de la «pure jouissance passive de l'objettélévision » (p. 391).

12. Voir en matière de réception des textes: R. Charrier, L'Ordre des livres. Lecteurs, auteurs, bibliothèques en Europe entre XIV et XVIII ${ }^{e}$ siècles, Aix-en-Provence, Alinéa, 1992 et B. Lahire, La Raison des plus faibles. Rapport au travail, écritures domestiques et lectures en milieux populaires, Lille, Presses universitaires de Lille, 1993. Concernant la réception des images, voir J.-C. Passeron, "L'usage faible des images. Enquêtes sur la réception de la peinture", in Le Raisonnement sociologique. L'espace non-poppérien du raisonnement naturel, Paris, Nathan, 1991, p. 257-288.

13. O. Schwartz, op. cit., p. 382. Quelques pages plus loin, les images de la télévision sont interprétées comme un des éléments du décor d'un appartement ouvrier surchargé d'objets : « Le logement de René et Hélène est littéralement pléthorique : une centaine de plantes, deux chiens, des poissons, des oiseaux, des poupées, des photos, la télévision qui marche en permanence même si on ne la regarde pas, tout cela voisine dans un désordre doué de sens, fait pour produire du plein et de la couleur, forme de défense contre la frustration et contre l'enfermement sans perspectives » (p. 388-389).

14. C. Lévi-Strauss, «Leçon d'écriture», chap. XXVIII, Tristes tropiques, Paris, Plon, 1955, p. 337-349. Toutes les citations entre guillemets sont tirées de ces pages.

15. Par exemple, d'autres passages de Tristes tropiques comme de la thèse sur les Nambikwara montrent au contraire une société d'avant l'écriture marquée, à sa façon et selon des formes spécifiques, par les hiérarchies et la violence.

16. J. Derrida, De la grammatologie, Paris, Éd. de Minuit, 1967, p. 184.

17. On pourrait objecter à l'analyse menée ici que Tristes tropiques n'est pas un ouvrage qui appartient au genre anthropologique-scientifique, mais plutôt aux carnets de voyage. Mais, d'une part, la même description ethnographique (sans le commentaire théorique) peut se lire dans la thèse soutenue en 1948 par l'auteur sur La vie familiale et sociale des Indiens Nambikwara et, d'autre part, Lévi-Strauss a eu l'occasion à maintes reprises de répéter son hypothèse concernant l'écriture (dans Anthropologie structurale en 1958 et dans ses Entretiens avec Georges Charbonnier en 1961), allant même jusqu'à parler d'une «théorie marxiste de l'écriture ».

18. P. Bourdieu, Le Sens pratique, Paris, Éd. de Minuit, 1980, p. 58.

19. P. Veyne, "Propagande, expression, roi, image, idole, oracle", L'Homme, XXX (2), 1990, p. 7-26.

20. Cf. aussi L. Marin, «Visibilité et lisibilité de l'histoire. À propos des dessins de la colonne Trajane », De la représentation, Paris, Gallimard/Seuil, 1994, p. 219-234.

21. J. Svenbro, Phrasikleia. Anthropologie de la lecture en Grèce ancienne, Paris, La Découverte, 1988, p. 54 .

22. Ibid., p. 212-213.

23. F. Dupont, L'invention de la littérature. De l'ivresse grecque au livre latin, Paris, La Découverte, 1994.

24. On peut, de ce point de vue, lire La Raison graphique. La domestication de la pensée sauvage (Paris, Ed. de Minuit, 1980) de J. Goody comme un texte de réflexion épistémologique sur les opérations savantes (scripturales et graphiques) et, notamment, sur celles du structuralisme. Nous nous permettons de renvoyer également au chapitre I ( Cultures écrites et cultures orales ») de notre ouvrage Culture écrite et inégalités scolaires. Sociologie de l'«échec scolaire » à l'école primaire, Lyon, Presses universitaires de Lyon, 1993, p. 7-41. 
25. B. Lahire, «Linguistiquc/écrirure/pédagogie : champs de pertinence et transferts illégaux », L'Homme et la Société, Revue internationale de recherches et de synthèses en sciences sociales, 101, 3/1991, p. 109-119.

26. J.-C. Passeron, Le Raisonnement sociologique..., op. cit., p. 284.

27. B. Lahire, "La variation des contextes en sciences sociales. Remarques épistémologiques ", Annales, Histoire, sciences sociales, L I, 1996, p. 381-407.

28. O. Ducrot, Le Dire et le dit, Paris, Éd. de Minuit, 1984, p. 124.

29. P. Bourdieu, La Distinction. Critique sociale du jugement, Paris, Éd. de Minuit, 1979, p. 211. C'est l'auteur qui souligne.

30. Ibid., p. 214.

31. «Le goût, propension et aptitude à l'appropriation (matérielle et/ou symbolique) d'une classe déterminée d'objets ou de pratiques classés et classants, est la formule génératrice qui est au principe du style de vie, ensemble unitaire de préférences distinctives qui expriment, dans la logique spécifique de chacun des sous-espaces symboliques, mobilier, vêtement, langage ou hexis corporelle, la même intention expressive. Chaque dimension du style de vie "symbolise avec" les autres, comme disait Leibniz, et les symbolise : la vision du monde d'un vieil artisan ébéniste, sa manière de gérer son budget, son temps ou son corps, son usage du langage et ses choix vestimentaires, sont entiers présents dans son éthique du travail scrupuleux et impeccable, du soigné, du fignolé, du fini et son esthétique du travail qui lui fait mesurer la beauté de ses produits au soin et à la patience qu'ils ont demandés.» P. Bourdieu, La Distinction..., ibid., p. 193-194.

32. Cf. B. Lahire, Tableaux de familles. Heurs et malheurs scolaires en milieux populaires, Paris, Gallimard/Seuil, 1995.

33. Pour prendre l'exemple d'un auteur cher à Pierre Bourdieu et qui pourrait bien avoir été l'inspirateur de l'écriture de certains passages.

34. P. Bourdieu, La Distinction..., op. cit., respectivement p. 194, 197 et 211 . On remarquera au passage la formule: «Il serait facile de montrer...» qui suit un: «Cette opposition se retrouverait... ». Voir aussi : « Il faudrait soumettre à une comparaison systématique... » (p. 215).

35. Mais, encore une fois, on ne sait pas si des observations ont été faites et, si c'est le cas, quels en sont le volume et l'étendue, dans quelles conditions elles ont été réalisées, etc.

36. P. Bourdieu, La Distinction..., op. cit., p. 204.

37. R. Barthes, « L'effet de réel », Communications, 11,1968.

38. Si le romancier " recourt à l'observation ou à la documentation », il les « biaise toujours en les pliant au seul souci de la "littérarité" des "effets de réels" ». À ce moment-là, l'auteur fait davantage « usage d'un savoir-faire littéraire dans le "faire-croire" romanesque » qu'un "usage contraignant [...] des méthodes d'observation, d'enquête et de traitement des données, mises au service d'une démarche ne visant qu'au renforcement des preuves et au contrôle de l'interprétation ». Cf. J.-C. Passeron, R. Moulin et P. Veyne, «Entretien avec Jean-Claude Passeron : un itinéraire de sociologue ", Revue européenne des sciences sociales, t. XXXIV, 103, 1996, p. 293.

39. P. Bourdieu, La Distinction..., op. cit., p. 217-218. C'est nous qui soulignons.

40. Cf. P. Bourdieu, ibid., p. 164-165; 186-187; 212-213; $223 ; 434 ; 439 ; 449$; 529 ; et l'on remarquera au passage que les classes populaires sont davantage photographiées et moins longuement analysées discursivement que les autres classes ( Le choix du nécessaire », chapitre consacré aux classes populaires, comporte 28 pages dont 4 de photographies ; « La bonne volonté culturelle ", à propos des petites bourgeoisies, 56 pages sans aucune photographie et « Le sens de la distinction", chapitre sur les bourgeoisies, 71 pages dont 4 de photographies (mais exclusivement des photographies de presse).

41. II existe un écart considérable, que tout lecteur peut expérimenter, entre l'impression de compréhension spontanée éprouvée en regardant les photographies - qui paraissent être de 
bonnes exemplifications des analyses et des descriptions que l'on a lues par ailleurs - et le sentiment de difficulté et d'inévidence que provoque l'effort d'explicitation des traits pertinents de description de représentations photographiques non commentées.

42. « Le bon vivant n'est pas seulement celui qui aime à bien manger et bien boire. Il est celui qui sait entrer dans la relation généreuse et familière, c'est-à-dire à la fois simple et libre que le boire et le manger en commun favorisent et symbolisent, et où s'anéantissent les retenues, les réticences, les réserves qui manifestent la distance par le refus de se mêler et de se laisseraller. », P. Bourdieu, La Distinction..., op. cit., p. 200.

43. P. Bourdieu, ibid., p. 218.

\section{RÉSUMÉS}

Dans le champ des sciences sociales, toutes les interprétations ne se valent pas. Le travail interprétatif s'y distingue en effet de l'herméneutique libre, c'est-à-dire des interprétations sauvages, empiriquement non contraintes. Une partie des interprétations imprudentes ou inadéquates est constituée par des surinterprétations. On peut en distinguer trois types : celles dues aux décrochages interprétatifs par rapport aux situations interprétées (type 1); celles produites par le décalage non objectivé, non contrôlé et non corrigé entre la situation du chercheur face aux matériaux étudiés et la situation des enquêtés (type 2) ; celles engendrées par la combinaison de deux éléments : la sur-abondance de preuves pour prouver la pertinence du modèle théorique utilisé et la nature «littéraire» (par emploi de procédés d'écriture) de la production d'effets de preuve avec la profusion d'exemples sur mesure, opposés aux exemples et contre-exemples engendrés ordinairement par l'enquête empirique (type 3). Pour aller au-delà des analyses poussives et autres descriptions plates en régime de sous-interprétation, toute interprétation sociologique pertinente pourrait être définie comme une surinterprétation contrôlée.

In the field of social sciences, all interpretations are not of equal worth. Indeed, the interpretative work in social sciences is distinguishable from free hermeneutics, that is to say, from wild interpretations, empirically unconstrained. A part of these imprudent or inadequate interpretations is constituted by what one could call overinterpretations. Three kinds can be distinguished: those due to interpretative break of contact with reference to interpretative situations (type 1); those produced by the non objectivised, uncontrolled and incorrected lag between the position of the researcher confronted with the material and the state of the inquiry (type 2) ; those engendered by the combination of two elements: over abundance of evidence brought to prove the relevance of the theoretical model and the "literary" nature (by use of processes of writing) of the production of results of proof with the profusion of tailored examples, opposed to examples and counter-examples engendered ordinarily by the empirical inquiry (type 3). To go beyond short-winded analysis and other flat descriptions which remain in the sphere of overinterpretation, every pertinent sociological interpretation can after all be defined as a controlled overinterpretation. 